\title{
Petrophysical property and seismic attribute relationships in pre-salt carbonates of the South Atlantic
}

Pedro Bevilacqua M.P. Vasconcellos* (Petrobras/Heriot-Watt University); Alexandre R. Maul (Petrobras/Heriot-Watt University); Patrick W.M. Corbett (Heriot-Watt University)

Copyright 2021, SBGf - Sociedade Brasileira de Geofísica.

This paper was prepared for presentation during the $17^{\text {th }}$ International Congress of the Brazilian Geophysical Society held in Rio de Janeiro, Brazil, 16-19 August 2021

Contents of this paper were reviewed by the Technical Committee of the $17^{\text {th }}$ International Congress of the Brazilian Geophysical Society and do not necessarily represent any position of the SBGf, its officers or members. Electronic reproduction or storage of any part of this paper for commercial purposes without the written consent of the Brazilian Geophysical Society is prohibited.

\begin{abstract}
Petrophysical property distribution using sparse well-log information and seismic attributes as continuous property when presenting strong correlations is a standard practice, building models to plan for oil-fields activities. However, the simple methods commonly used can break down due to the seismic data quality, lack of feasible data correlation, and other issues. This work explores what are some of these limitations and provides a possible solution. The area is located in the Santos Basin, Brazilian Coast, and is composed of a pre-stack depth migrated (PSDM) seismic volume, covering approximately $200 \mathrm{~km}^{2}$, and logs from 11 drilled wells. The main objective is an attempt to integrate seismic attributes as a geostatistical drift parameter together with well-log petrophysical properties to build reliable geological models. We divided the wells into two groups, the first having seven wells, named as training dataset, and the second containing four wells named as validating dataset. We took the training dataset to create the models and used the other four to perform a blind test aiming to validate the built models. Our initial approach regarding the seismic data was to choose a number of instantaneous amplitude attributes, all available on commercial software. Here we opted to consider attributes combining amplitude, frequency, and phase components of the seismic traces. Further, still using the same commercial software, it was possible to test several cross-correlations between petrophysical properties and seismic attributes in order to get ways to propagate the well information having the seismic attribute as spatial control, giving more geological confidence to the built 3D model. The attributes used in this project were the relative acoustic impedance (RAI) and the envelope (total instantaneous energy of the entire analytical trace) that have demonstrated the best correlations when observing the petrophysical information. In order to limit the analysis to a consistent stratigraphic interval, we generated attribute maps of average values within a window $40 \mathrm{~m}$ below the mapped stratigraphic horizons. The criteria for using this window was based on the seismic vertical resolution, which is also close to the average thickness of the stratigraphic cycles of this area. The main reason to create maps starting from the top of the stratigraphic unit (SU) was to try to compare intervals of reservoir rocks related to the same stratigraphic cycle. The median of total porosity in the intervals of each well and the attributes show a very high degree of cross-correlation level (r2>7.5). Later we created 3D porosity models using a Sequential Gaussian Simulation (SGS) algorithm and collocated cokriging in order to test with the validating dataset. The resulted porosity model indicates significant areal variations, which are in good agreement with the well-logs. We must carefully consider the matches over the different sectors of the field, as well as the different geological contexts. Although a relatively simple study, apart from some ambiguities, especially for the seismic instantaneous attribute response, we believe this method feasible and with few adjustments valuable for predict the reservoir characteristics in future well placement considerations.
\end{abstract}

